\title{
Magnetic liquid marbles, their manipulation and application in optical probing
}

\author{
Yan Zhao $\cdot$ Zhiguang Xu $\cdot$ Marzieh Parhizkar • \\ Jian Fang $\cdot$ Xungai Wang $\cdot$ Tong Lin
}

Received: 14 February 2012/ Accepted: 27 March 2012/Published online: 10 April 2012

(C) The Author(s) 2012. This article is published with open access at Springerlink.com

\begin{abstract}
Magnetic liquid marbles, an encapsulation of liquid droplet with hydrophobic magnetic particles, show remarkable responsiveness to external magnetic force and great potential to be used as a discrete droplet microfluidic system. In this study, we presented the manipulation of a magnetic liquid marble under an external magnetic field and calculated the maximum frictional force, the magnetic force required for actuating the liquid marbles and the effective surface tension of the magnetic liquid marble, as well as the threshold volume for the transition from quasispherical to puddle-like shape. By taking advantage of the unique feature of being opened and closed reversibly, we have proven the encapsulated droplets can be detected optically with a reflection-mode probe. Combining the open-close and optical detection also enables to probe chemical reactions taking place within liquid marbles. These remarkable features offer a simple yet powerful alternative to conventional discrete microfluidic systems and may have wide applications in biomedical and drug discovery.
\end{abstract}

Keywords Liquid marble - Microfluidics . Magnetic nanoparticle $\cdot$ Microreactor . Droplet

\section{Introduction}

Liquid marbles are liquid droplet-powder encapsulations consisting of a small amount of liquid being covered with

Y. Zhao - Z. Xu · M. Parhizkar · J. Fang - X. Wang .

T. Lin $(\square)$

Australian Future Fibres Research and Innovation Centre,

Deakin University, Geelong, VIC 3217, Australia

e-mail: tong.lin@deakin.edu.au hydrophobic powders. They were first reported in 2001 (Aussillous and Quere 2001), and since then growing attention has been paid toward this interesting material (Bormashenko 2011; McHale and Newton 2011). Liquid marbles are formed due to the spontaneous attachment of hydrophobic particles on the liquid/air interface when a small volume of liquid is rolled around. The mechanism behind the liquid marble formation is thought to be the minimized overall surface energy when hydrophobic particles attach on droplet surface (McHale et al. 2007). When the particle is in spherical shape, its attachment on the liquid surface changes the air/solid interfacial area by $-2 \pi R^{2}\left(1+\cos \theta_{e}\right)$, where $R$ is the radius of the particle and $\theta_{e}$ is the equilibrium contact angle. The changes in the liquid/solid and air/liquid interfacial areas are $2 \pi R^{2}(1+$ $\left.\cos \theta_{e}\right)$ and $-\pi R^{2} \sin ^{2} \theta_{e}$, respectively. Therefore, the net change in the surface energy is

$\Delta F=2 \pi R^{2}\left(1+\cos \theta_{e}\right)\left(\gamma_{s l}-\gamma_{s v}\right)-\pi R^{2} \sin ^{2} \theta_{e} \gamma_{l v}$

where $\gamma_{i j}$ is the interfacial tension $(i, j=s, l$ and $v)$. According to the Young's law: $\gamma_{s v}-\gamma_{s l}=\gamma_{l v} \cos \theta_{e}$, Eq. (1) can be re-written as

$\Delta F=-\gamma_{l v} \pi R^{2}\left(1+\cos \theta_{e}\right)^{2}$

Since this change is always negative in value, the attachment of hydrophobic particles on the air/liquid interface should always be favorable to stabilize the droplet.

Once a liquid marble is formed, the liquid droplet is completely covered with a thin powdery layer, and this separates the liquid from the outside solid substrate on which the liquid marble sits. For small droplets, liquid marbles adopt a quasi-spherical shape on smooth substrates and are considered as perfect non-wetting systems. The small contact of a liquid marble with the substrate makes it 
easy to move without leaking the liquid, and hence, no contamination. This feature provides liquid marbles with great potential for discrete droplet microfluidics.

A diversity of powders has been used to produce liquid marbles, including natural lycopodium, poly(tetrafluoroethylene), polyethylene, hydrophobized copper, graphite, and silica aerogels (Bormashenko 2011). The particle size of the powders can be varied from tens of nanometers to several micrometers. Apart from water and aqueous solutions, some low-surfacetension solvents, including diiodomethane, hexadecane, octane, toluene, and ionic liquids, have also been reported to form liquid marbles, and the formation of such liquid marbles requires highly liquid-repellent particles such as perfluorinated oligomeric tetrafluoroethylene (OTFE) particles (Gao and McCarthy 2007) or fluorinated decyl polyhedral oligomeric silsesquioxane (FD-POSS) powders (Xue et al. 2010).

Discrete droplets have shown potential to be used as droplet microfluidics, a completely different microfluidic system to the conventional channel-based microfluidics (Abdelgawad and Wheeler 2009). Compared with the channel-based fluidics, this relatively new microfluidic system is advantageous in reducing the sample size and cross mass-transport between samples, straightforward to use and easy integration with other components (i.e., the lab-on-chip concept). Discrete droplet microfluidics are conventionally formed by suspending aqueous droplets in an oil phase. The droplets can be actuated by an external electric (Srinivasan et al. 2004) or magnetic field (Lehmann et al. 2006; Dorvee et al. 2004), or an acoustic action (Guttenberg et al. 2005). However, these oil-immersed systems met problems with poor ability to communicate with the outside and potential for liquid-liquid extraction of analytes into the surrounding oil (Abdelgawad et al. 2008).

Recently, the development of lotus-inspired superhydrophobic surfaces (McHale et al. 2004; Callies and Quere 2005; Feng et al. 2002) has led to the possibility to eliminate oil phase from the discrete fluidic systems. When a substrate has a superhydrophobic surface, water droplets can sit on the surface to form nearly spherical balls. To manipulate the droplets, paramagnetic micro/nanoparticles are often incorporated into the droplets to impart them with a magnetic responsive ability (Egatz-Gomez et al. 2006, 2007; Lindsay et al. 2007; Garcia et al. 2007; Guo et al. 2006; Schneider et al. 2008; Bormashenko et al. 2008). A guiding wire that directly contacts with the droplet was also employed to direct the droplet movement (Mumm et al. 2009; Yoon and You 2008). In addition, surface tensioninduced pressure gradient was used to drive the droplet movement on a superhydrophobic surface (Hong and Pan 2011). Although the droplet microfluidics based on superhydrophobic surfaces can overcome some drawbacks facing to oil-immersed systems, it has an issue in the fast evaporation of liquid from the droplets.
Liquid marbles have been reported to be able to reduce the evaporation of liquid because of the coverage of hydrophobic particles (Dandan and Erbil 2009). This makes them promising for applications in discrete droplet microfluidics. Recently, we have found that when magnetic particles were used to form liquid marbles (Zhao et al. 2010; Xue et al. 2010), the powdery shell on liquid marbles can be opened and closed reversibly with a magnetic force. The magnetic manipulation of liquid marbles offers a great opportunity for the liquid inside to be detected by external optical equipment on-demand. However, optical probing of magnetic liquid marbles has not been demonstrated in the research literature.

In this study, we demonstrate the optical detection of the liquid marbles for possible application as a new discrete microfluidic system. The maximum frictional force and the external magnetic force required for magnetically actuating the liquid marbles, the effective surface tension of the magnetic liquid marble, and the threshold volume for the transition from quasi-spherical to puddle-like shape are calculated.

\section{Experimental}

\subsection{Synthesis of $\mathrm{Fe}_{3} \mathrm{O}_{4}$ nanoparticles}

Superhydrophobic $\mathrm{Fe}_{3} \mathrm{O}_{4}$ nanoparticles were prepared by co-precipitation of ferric and ferrous ions and simultaneous hydrolysis of tridecafluorooctyltriethoxysilane in an alkaline solution. Briefly, $1.5 \mathrm{M}$ aqueous $\mathrm{NH}_{4} \mathrm{OH}$ solution was added dropwise to $200 \mathrm{ml}$ water/ethanol solution $(4: 1, \mathrm{v} / \mathrm{v})$ containing $\mathrm{FeCl}_{3} \cdot 6 \mathrm{H}_{2} \mathrm{O}(0.85 \mathrm{~g}, 3.14 \mathrm{mmol}), \mathrm{FeCl}_{2} \cdot 4 \mathrm{H}_{2} \mathrm{O}$ $(0.30 \mathrm{~g}, 1.51 \mathrm{mmol})$ and tridecafluorooctyltriethoxysilane $(0.20 \mathrm{ml}, 5.23 \mathrm{mmol})$ under continuous nitrogen purging and vigorous magnetic stirring until $\mathrm{pH}=8$. After stirring for $24 \mathrm{~h}$, the resulting precipitate (magnetite $\mathrm{Fe}_{3} \mathrm{O}_{4}$ ) was isolated from the solution with a bar magnet, washed with water/ethanol $(4: 1, \mathrm{v} / \mathrm{v})$ mixture for three times, and dried at $60{ }^{\circ} \mathrm{C}$.

\subsection{Manipulation of magnetic liquid marbles}

Magnetic liquid marbles were formed by rolling a water droplet on a layer of superhydrophobic $\mathrm{Fe}_{3} \mathrm{O}_{4}$ nanoparticles. Magnetic manipulation of liquid marbles was carried out with permanent neodymium cylinder magnets $10 \mathrm{~mm}$ in diameter and $12 \mathrm{~mm}$ in length (AMF magnetics).

\subsection{Characterizations}

Water contact angles were measured using a CAM101 video camera-based contact angle measurement system 
(KSV Instruments Ltd, Finland). Transmission electron microscopy (TEM) image was obtained using a FEI Tecnai F30 Cryo-transmission electron microscope. X-ray powder diffraction (XRD) pattern was recorded on a Philips 1,140/ 90 diffractometer using $\mathrm{Cu} \mathrm{K} \alpha(\lambda=1.54 \AA)$ radiation. Magnetic properties of the nanoparticles were measured with a Quantum Design MPMS-5 DC-SQUID (superconducting quantum interference device) susceptometer. UVvisible absorption spectra of bulk liquids were recorded using a Varian Cary 3 spectrophotometer. Magnetic field intensity was measured using a PASCO magnetic field sensor (PS-2162). The directed movement of liquid marbles was recorded with a Dino-Lite digital microscope (AM313).

For optical detection, a reflection/backscattering probe consisting of a central optical read fiber surrounded by six light-source optical fibers was used (Ocean Optics, R4007-UV/VIS). The central read fiber collects the backscattered light and transfers the signal to a miniature USB4000 spectrometer. The light source was a DH2000-BAL device that combines deuterium and tungsten halogen light for an output in the wavelength range of 230-2,000 nm. All these devices were purchased from Ocean Optics (Dunedin, FL).

\section{Results and discussion}

\subsection{Superhydrophobic $\mathrm{Fe}_{3} \mathrm{O}_{4}$ nanoparticles}

Figure 1a shows the TEM image of the as-synthesized $\mathrm{Fe}_{3} \mathrm{O}_{4}$ nanoparticles. They looked roughly spherical in shapes and had an average size of $10.2 \pm 2.7 \mathrm{~nm}$. Figure $1 \mathrm{~b}$ shows the XRD pattern of the $\mathrm{Fe}_{3} \mathrm{O}_{4}$ nanoparticles. The diffraction peaks at $2 \theta=18.6,30.4,35.7,43.2,53.7$, $57.3,62.8,71.4$, and $74.5^{\circ}$ can be indexed, respectively, to the (111), (220), (311), (400), (422), (511), (440), (620), and (533) planes in a face-centered cubic $\mathrm{Fe}_{3} \mathrm{O}_{4}$. The crystallite size $(D)$ can be calculated using Debye-Scherrer equation (Huang et al. 2011):
$D=\frac{K \lambda}{\beta \cos \theta}$

where $K$ is the shape factor (generally taken as 0.9 ), $\lambda$ is the $\mathrm{X}$-ray wavelength $(0.15418 \mathrm{~nm}$ for $\mathrm{Cu}-\mathrm{K} \alpha$ radiation $), \beta$ is the full-width at half-maximum (fwhm) of the diffraction peak, and $\theta$ is the Bragg diffraction angle. By using the diffraction peak (311), the crystallite size was calculated to be $8.6 \mathrm{~nm}$, which is close to the value determined by the TEM image.

Figure 2 shows the magnetic properties of the $\mathrm{Fe}_{3} \mathrm{O}_{4}$ nanoparticles. The magnetization was measured as a function of temperature in the range of $2-300 \mathrm{~K}$ in the applied field of $0.01 \mathrm{~T}$ using field cooling and zero-field cooling procedure. As shown in Fig. 2a, the blocking temperature of the nanoparticles is $98 \mathrm{~K}$, suggesting a particle size of around $12 \mathrm{~nm}$ (Park et al. 2004). The hysteresis loop shown in Fig. 2b indicates the typical superparamagnetic behavior of the $\mathrm{Fe}_{3} \mathrm{O}_{4}$ nanoparticles. They only showed magnetic characteristics under an external magnetic field, but did not retain any magnetization once the external magnetic field was absent. At $295 \mathrm{~K}$, the saturation magnetization was $32 \mathrm{~A} \mathrm{~m}^{2} / \mathrm{kg}$ at $5 \mathrm{~T}$. Figure $3 \mathrm{a}$ shows the profile of a water droplet placed on a compact bed of the $\mathrm{Fe}_{3} \mathrm{O}_{4}$ nanoparticles. The contact angle was measured to be $156.5^{\circ} \pm 3.2^{\circ}$, showing a superhydrophobic property.

\subsection{Magnetic liquid marbles}

Liquid marbles were formed by rolling a water droplet on the superhydrophobic $\mathrm{Fe}_{3} \mathrm{O}_{4}$ nanoparticles. As illustrated in Fig. 3b, the $\mathrm{Fe}_{3} \mathrm{O}_{4}$ nanoparticles spontaneously self-organized on the droplet surface to form a uniform layer covering on the entire droplet. This self-attachment of particles to liquid droplet surface was attributed to the minimization of the surface free energy (Aussillous and Quere 2001; McHale et al. 2007). Figure 3c shows a photograph of the formed liquid marble on a glass substrate.
Fig. 1 a TEM image and b XRD pattern of the as-synthesized $\mathrm{Fe}_{3} \mathrm{O}_{4}$ nanoparticles (a)

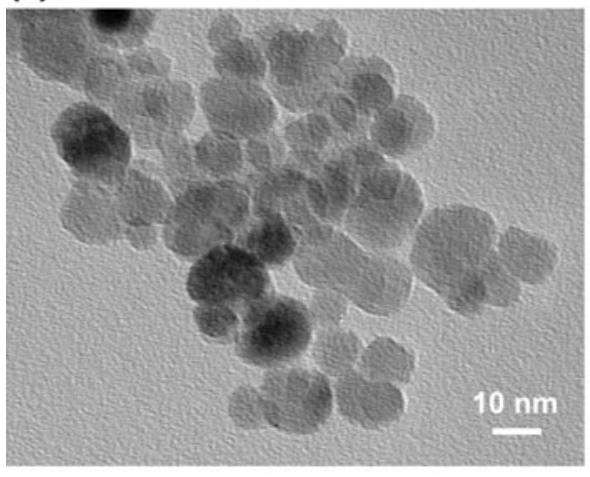

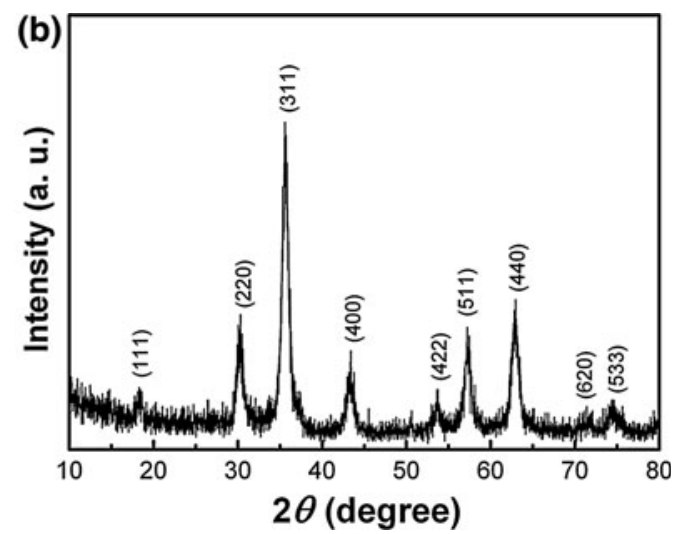


Fig. 2 Magnetic properties of the $\mathrm{Fe}_{3} \mathrm{O}_{4}$ nanoparticles. a Zerofield-cooling and field-cooling (ZFC-FC) curves measured with the field of $0.01 \mathrm{~T}$, and b magnetization curve measured at $295 \mathrm{~K}$
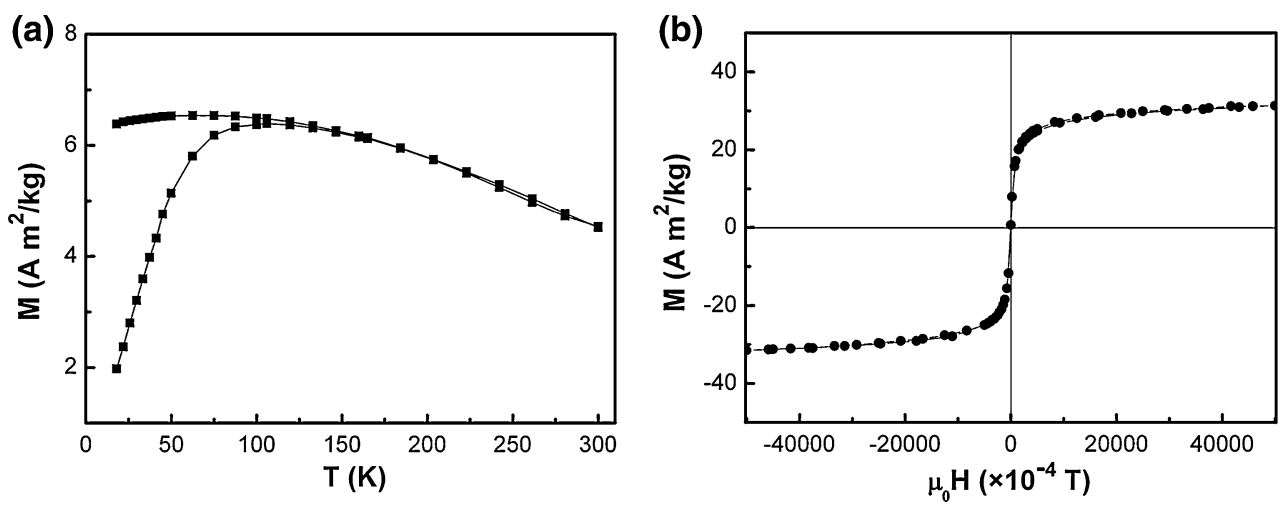

(a)

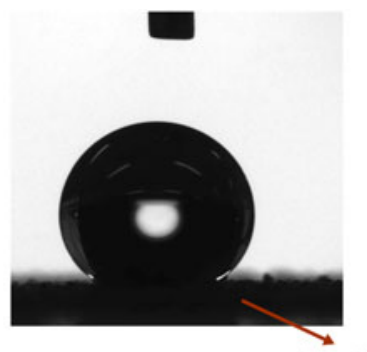

(c)

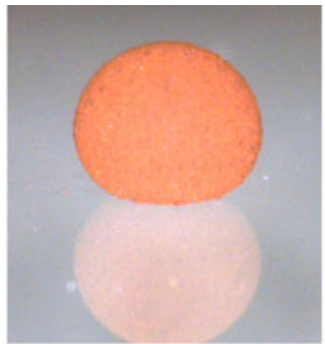

$\mathrm{Fe}_{3} \mathrm{O}_{4}$ nanoparticles

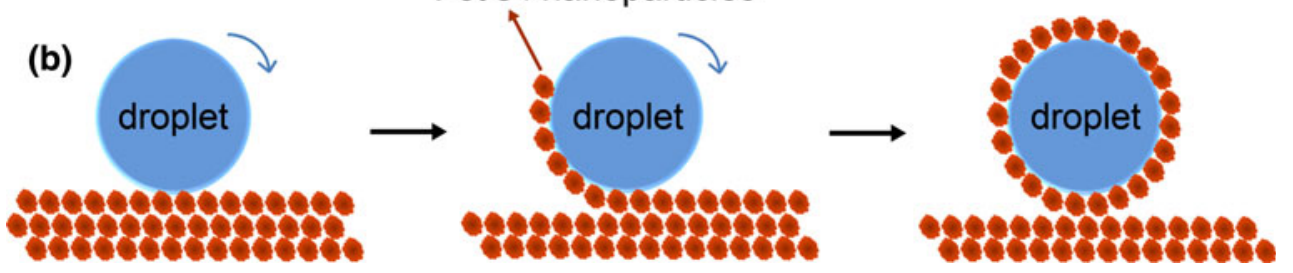

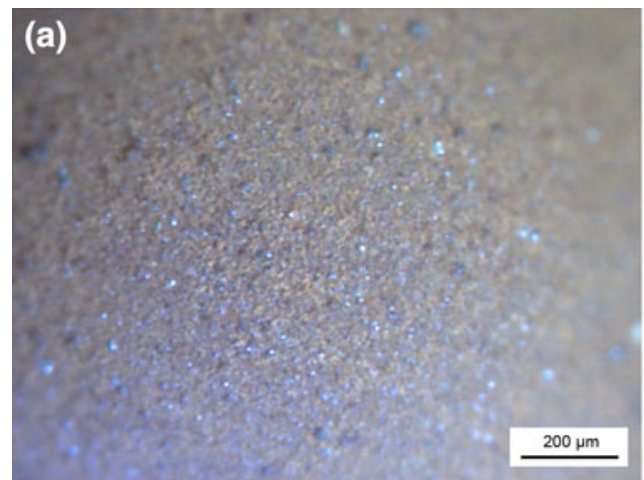

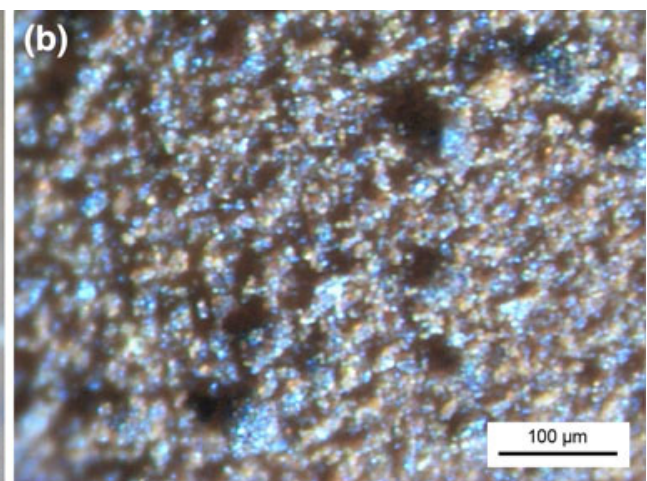

Fig. 4 Optical microscopy images of the outside surface of a liquid marble prepared with superhydrophobic $\mathrm{Fe}_{3} \mathrm{O}_{4}$ nanoparticles. The blue spots are water areas, indicating the voids existing in the nanoparticle coating (colour figure online)

The packing of nanoparticle powders at the water/air interface was observed using an optical microscope. Figure 4 shows the microscopy images of the surface of a liquid marble, revealing that although the powders are packed well at the droplet surface there are still some voids existing in the coating because of the irregular shape of the powders. Owing to the hydrophobicity of the nanoparticles, however, water cannot penetrate these voids. It is important to stress here that the loosely packed coating is not rigid, but behaves like a flexible skin, and can deform itself to follow the contour of the droplet. This makes liquid marble a soft but elastic solid that can bounce and roll without leaking of the liquid.

The shape of a liquid marble is determined by the balance between the gravity and the surface tension. As shown in Fig. 5, the liquid marbles take a quasi-spherical shape for small droplets, but with the increase in volume, the marbles are deformed more strongly by gravity and finally 
become puddle-shaped. It is known that the saturated value of the puddle height $\left(H_{\max }\right)$ is fixed to be twice of the capillary length (Aussillous and Quere 2001):

$\kappa^{-1}=\sqrt{\frac{\gamma}{\rho g}}$

where $\kappa^{-1}$ is the capillary length $\left(\kappa^{-1}\right.$ is $2.7 \mathrm{~mm}$ for water), $\gamma$ is the surface tension of the liquid marble, $\rho$ is the liquid density, and $g=9.81\left(\mathrm{~m} \mathrm{~s}^{-2}\right)$ is the acceleration due to gravity. The effective surface tension of the liquid marble can be calculated based on the $H_{\max }$ value:

$\gamma=\frac{\rho g H_{\max }^{2}}{4}$

The $H_{\max }$ value can be obtained by geometrical measurements of the height $(H)$ of a liquid marble as a function of its horizontal diameter $(D)$ with increasing its volume. Based on the results shown in Fig. 5, $H_{\max }$ is estimated to be $5 \mathrm{~mm}$, i.e., $\kappa^{-1}$ is $2.5 \mathrm{~mm}$ for our magnetic liquid marble. According to Eq. (5), the effective surface tension of the liquid marble is calculated to be $61 \mathrm{mN} / \mathrm{m}$. Compared with pure water $(\gamma=72 \mathrm{mN} / \mathrm{m})$, the reduced surface tension can be ascribed to the contribution of the superhydrophobic $\mathrm{Fe}_{3} \mathrm{O}_{4}$ nanoparticle coating. Usually, droplets with initial radius larger than $\kappa^{-1}$ are flattened by the action of gravity to form puddles (Quere and Aussillous 2002). In our case $\left(\kappa^{-1}=2.5 \mathrm{~mm}\right)$, liquid marbles with a volume $>65 \mu \mathrm{L}$ are considered to be in puddle-like shape. Quasi-spherical liquid marbles with a size of $10-20 \mu \mathrm{L}$ (droplet diameter 2.6-3.4 mm) were used for the following study on the magnetic manipulation and optical detection of liquid marbles.

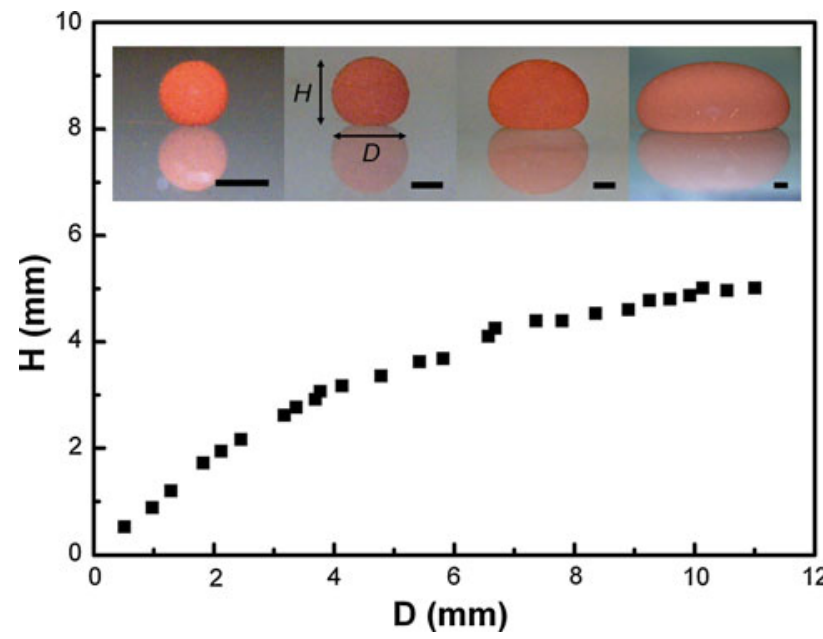

Fig. 5 Changes in the height $(H)$ of a liquid marble as a function of its horizontal diameter $(D)$ with increasing the volume. Inserts are photos showing the transition from nearly spherical to puddle-like shape as increasing the volume of the liquid marble (volume 1, 5, 45, and $340 \mu \mathrm{L}$, respectively). Scale bar $1 \mathrm{~mm}$

\subsection{Magnetic manipulation}

The magnetic liquid marble placed on a flat and smooth solid substrate had a contact angle close to $180^{\circ}$ and it can be moved effortlessly due to gravitational force once the substrate was slightly tilted. This means that the frictional force between the liquid marble and the substrate is very small. According to Amonton's law, the maximum frictional force can be calculated as

$F_{\text {max }}=m g \sin \alpha$

where $\alpha$ is the sliding angle of the liquid marble, and $m$ and $g$ are the mass of the liquid marble and the acceleration due to gravity, respectively. For a $15-\mu \mathrm{L}$ liquid marble, the mass of magnetic particles on the droplet was about $0.49 \mathrm{mg}$, and the sliding angle on glass substrate was about $1^{\circ}$. The maximum frictional force, i.e., the threshold value of an external applied force to move the liquid marble, was calculated to be around $2.65 \mu \mathrm{N}$.

Figure 6 a shows the geometry of a particle attached at the water/air interface of a liquid marble; any attempt to move it into the droplet or into the air causes a change in the actual contact angle, $\theta$, and an increase in the surface free energy (McHale and Newton 2011):

$\Delta F=\gamma_{l v} \pi R^{2}\left(\cos \theta_{e}-\cos \theta\right)^{2}$

Generally, the magnetic nanoparticles coated on the droplet do not show their magnetism because every magnetic moment of a magnetic domain has its own direction. Once an external inhomogeneous magnetic field is applied, however, all magnetic domains are regulated to have the same direction. As a result, a magnetic force is generated with the direction pointing to the maximum of the magnetic field gradient. Meanwhile, the change in $\theta$ causes an interfacial force acting on the droplet, thus actuating the droplet.

Figure $6 b$ shows the magnetic actuation of a liquid marble $(15 \mu \mathrm{L})$ on a glass surface. Upon moving a magnet slowly toward the liquid marble, the liquid marble started moving once the distance was close enough (about $16 \mathrm{~mm}$ ). In an applied magnetic field, the force acting on each magnetic nanoparticle can be calculated according to (Zhang et al. 2009):

$F_{\mathrm{mag}, \mathrm{NP}}=\frac{V \Delta \chi}{\mu_{0}}(\nabla B) B$

where $V$ is the volume $\left(\mathrm{m}^{3}\right)$ of nanoparticle, $\Delta \chi$ is the difference in magnetic susceptibilities between the nanoparticle and the surrounding medium (dimensionless), $\mu_{0}=4 \pi \times 10^{-7}\left(\mathrm{~T} \mathrm{~m} \mathrm{~A}^{-1}\right)$ is the permeability of vacuum, $B$ and $\nabla B$ are magnetic flux density (T) and magnetic field gradient $\left(\mathrm{T} \mathrm{m}^{-1}\right)$, respectively. The total magnetic force acting on a liquid marble can be calculated as 
Fig. 6 a Schematic diagram illustrating the magnetic force acting on a magnetic liquid marble and the geometry of a particle lying at the water/air interface. b Magnetic actuation of a liquid marble $(15 \mu \mathrm{L})$ on a glass surface by moving slowly a magnet toward the liquid marble until the marble starts to move. $\mathbf{c}$ The measured magnetic flux density as a function of the distance away from the magnet surface (a)
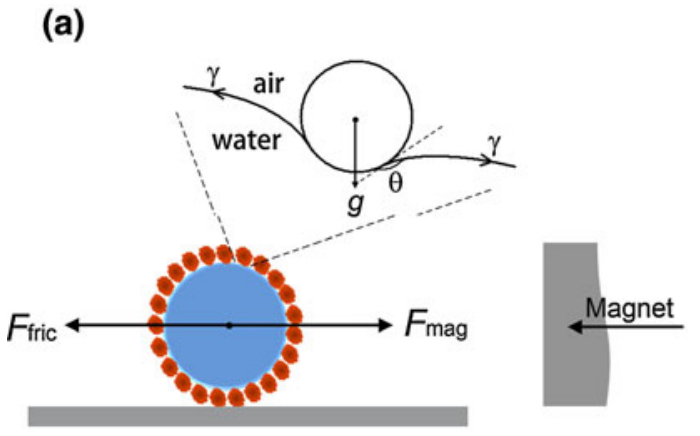

(b)

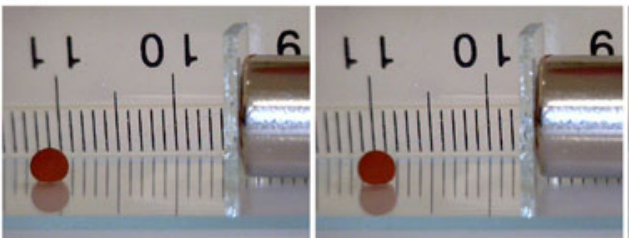

(c)

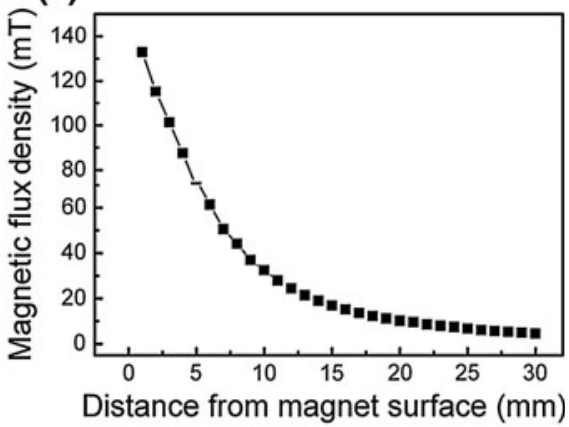

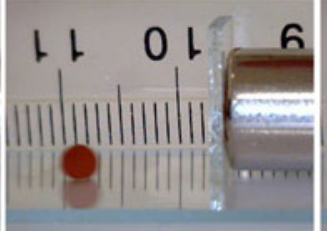

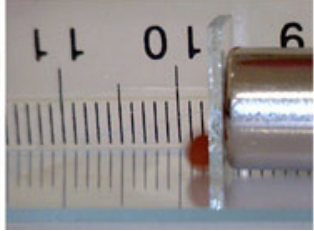

$F_{\mathrm{mag}}=\frac{m \Delta \chi}{\rho \mu_{0}}(\nabla B) B$

where $m$ is the total mass of magnetic particles on the droplet (4.9 $\times 10^{-7} \mathrm{~kg}$ for a $15 \mu \mathrm{L}$ liquid marble), and $\rho$ is the density of $\mathrm{Fe}_{3} \mathrm{O}_{4}$ nanoparticle $\left(5.18 \times 10^{3} \mathrm{~kg} \mathrm{~m}^{-3}\right)$.

In our case, the magnetic susceptibility of the $\mathrm{Fe}_{3} \mathrm{O}_{4}$ nanoparticle was calculated from the slope of a line fitted to the magnetization curve (Fig. $2 b$ ), to be 1.45 . The magnetic susceptibility of the surrounding medium can be neglected. Magnetic flux density was measured as a function of the distance away from the magnet surface. The results are shown in Fig. 6c. When the distance was $16 \mathrm{~mm}$, corresponding to the threshold value to actuate a $15 \mu \mathrm{L}$ liquid marble, the generated magnetic flux density and magnetic field gradient were $15.2 \mathrm{mT}$ and $1.76 \mathrm{~T} \mathrm{~m}^{-1}$, respectively. According to Eq. (9), the magnetic force acting on the liquid marble was calculated to be $2.92 \mu \mathrm{N}$, which agrees well with the value estimated by the tilting method mentioned above.

To smoothly move a magnetic liquid marble on a glass surface, the magnet was placed underneath the glass plate. The magnetic liquid marble can be moved along with the movement of the magnet (Fig. 7a). Herein, the magnetic liquid marble can also be actuated in a three-dimensional (3D) manner. As shown in Fig. 7b, a magnetic liquid marble was moved along the inner wall of a cylinder vessel by moving the magnet along the outer wall, which is not easy to be realized by other droplet manipulation techniques. This 3D transport will facilitate the generation of more compact, flexible, and topologically complex microfluidic systems.

Moreover, the magnetic liquid marbles can be opened and closed reversibly. As shown in Fig. 8, when the magnet was moved upward to approach the liquid marble, the $\mathrm{Fe}_{3} \mathrm{O}_{4}$ nanoparticles were pulled down toward the glass

(a)
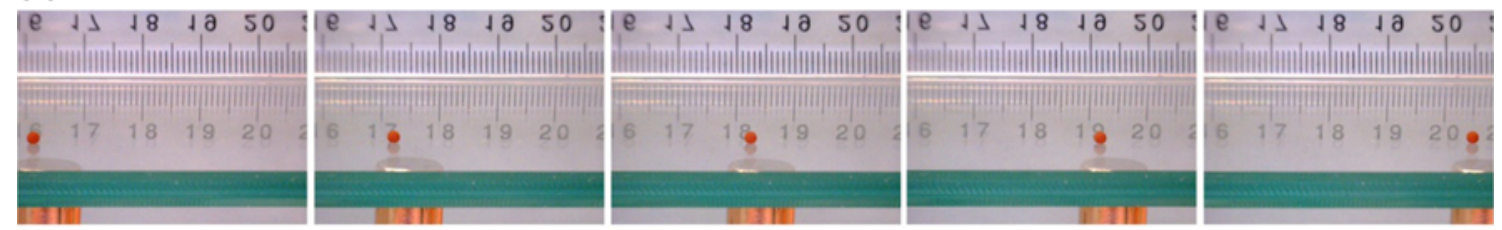

(b)
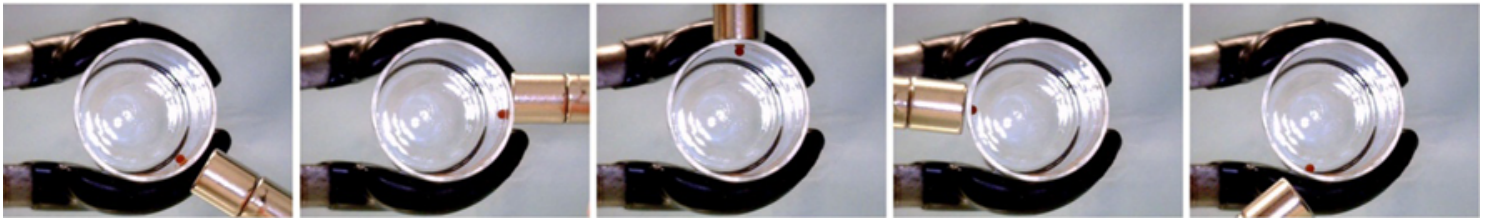

Fig. 7 Magnetically actuated movement. a Transport on a horizontal surface driven by a magnet positioned beneath the glass substrate. b Manipulation of a liquid marble on the inner wall of a cylinder vessel (23.5 mm diameter) 
(a)
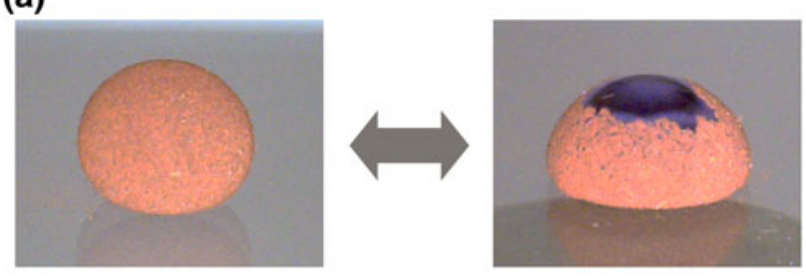

(b)
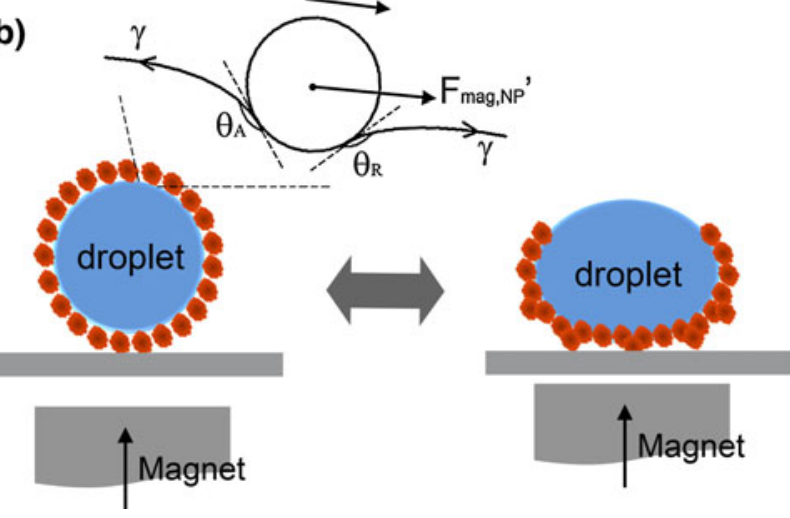

Fig. 8 a Reversible opening and closing of a magnetic liquid marble containing a blue-colored water droplet $(15 \mu \mathrm{L})$. b Schematic illustration of the opening process and the movement of a particle on liquid surface under the action of magnetic force (colour figure online)

surface, and the liquid marble was opened on the top. As long as the magnetic field existed, the liquid marble kept its open state. Once the magnet was removed, the $\mathrm{Fe}_{3} \mathrm{O}_{4}$ nanoparticles moved back, and the exposed liquid surface was covered with the magnetic powder again. The opening and closing of the liquid marble is reversible. This remarkable feature offers unique way to detect the liquid within the liquid marbles using external equipment.

The opening of a magnetic liquid marble can be understood by analyzing the force acting on the magnetic particles. A particle resting on the liquid surface is schematically illustrated in Fig. 8b. Suppose that the particle is just before sliding, the front and rear contact angles are close to the receding $\left(\theta_{\mathrm{R}}\right)$ and advancing $\left(\theta_{\mathrm{A}}\right)$ contact angles, respectively (Quere 1998). When the particle receives the magnetic force in the direction parallel to the liquid surface larger than $\pi r \gamma\left(\cos \theta_{\mathrm{R}}-\cos \theta_{\mathrm{A}}\right)$, it moves along the interface. Here, $r$ is the radius of the contact line, which is considered as circular, and $\gamma$ is the surface tension of the liquid.

\subsection{Optical probing}

To demonstrate the feasibility of optical probing, an optical detection technique was performed based on a reflectionmode. As shown in Fig. 9a, the liquid marble $(20 \mu \mathrm{L})$ is moved toward the optical probe with a magnet underneath (images 1-2). Upon reaching the destination, the magnet was moved to approach the liquid marble. Consequently, the top surface of the liquid marble was opened. An optical probe was therefore able to detect the liquid (image 3). After the detection, the liquid marble was closed by moving the magnet away to a distance that can keep the liquid marble in the closed state, but drove its movement to other location (images 4-5). A complete detection process consisting of droplet transport and detection was accomplished.

Figure $9 \mathrm{~b}$ shows the optical absorption curves of aqueous liquid marbles containing acid yellow 14, acid red 183 , or acid blue 25 . Their typical absorption peaks at 392,491 , and $622 \mathrm{~nm}$, respectively, were observed. For comparison, the optical absorption curves of the bulk liquid solutions detected with a normal UV-visible spectrophotometer were also shown in Fig. 9c, which are consistent with those obtained by the optical detection of liquid marbles. This result indicates the reliability of this optical detection method.
Fig. 9 Optical detection. a Sequence of frames from a video showing a magnetic liquid marble $(20 \mu \mathrm{L})$ being $(1-2)$ moved toward an optical probe, (3) opened for optical detection, (4-5) closed and moved away after the detection is completed under the action of a magnet. b Optical absorption curves of magnetic liquid marbles containing aqueous solutions of acid yellow 14 , acid red 183 , and acid blue 25 , respectively. c Optical absorption curves of bulk liquid solutions of acid yellow 14 , acid red 183, and acid blue 25 , respectively (a)

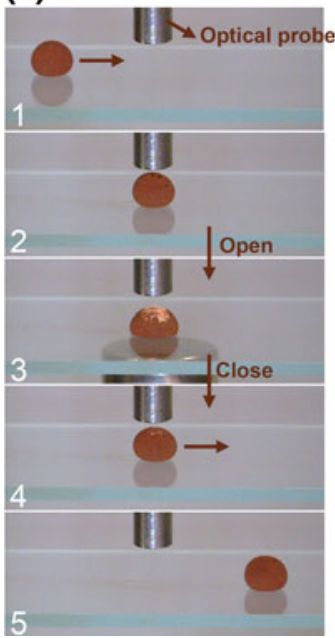

(b)

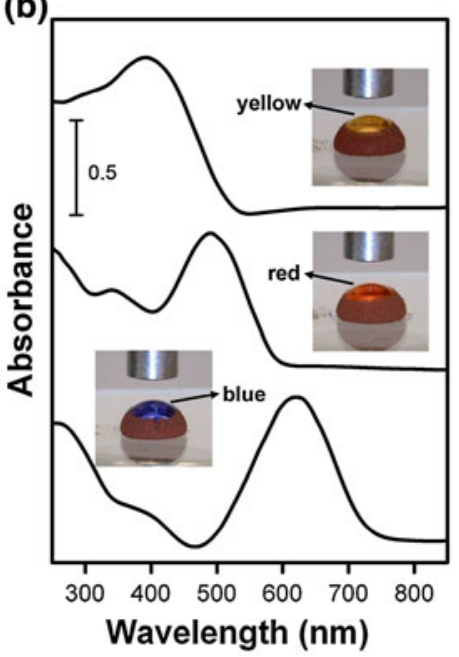

(c)

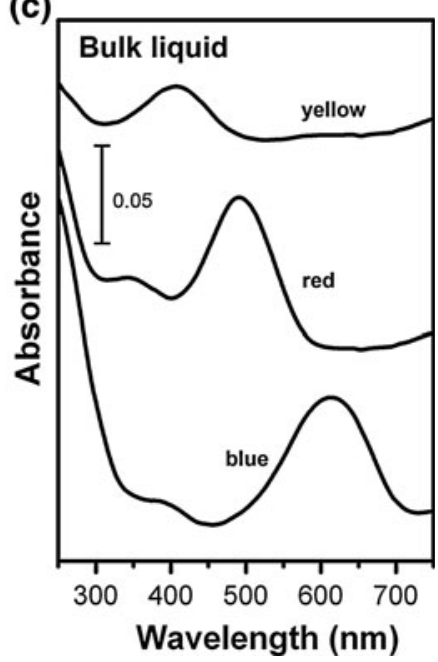




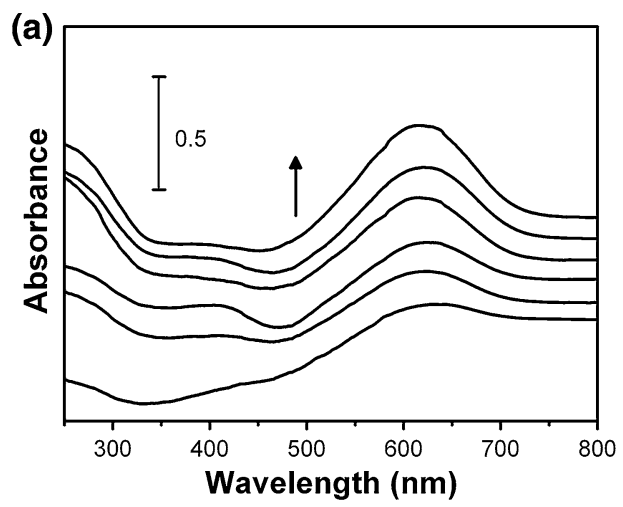

Fig. 10 Quantitative measurement. a Optical absorption curves of magnetic liquid marbles containing aqueous solutions of acid blue 25 with various concentrations (ascending along $y$-axis): 0.2, 0.4, 0.6, $0.8,1.0$, and $1.2 \times 10^{-5} \mathrm{M}$. b Calibration plot of absorption as a

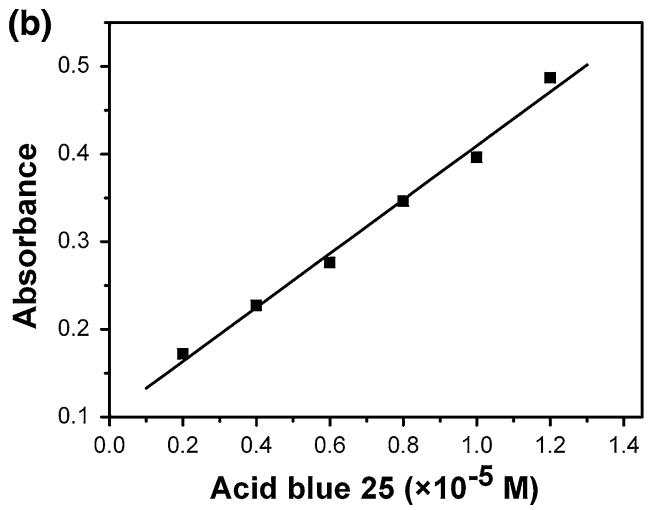

function of the concentration. The solid line represents a linear fit with regression equation: $y=0.1+0.3 x\left(R^{2}=0.9906, n=6\right)$. Liquid marble size $20 \mu \mathrm{L}$
Fig. 11 A chemical reaction and its optical detection. a Sequence of frames from a video demonstrating $(1-3)$ the optical detection of a magnetic liquid marble $(15 \mu \mathrm{L})$

containing $\mathrm{Fe}^{3+}$ solution (10 $\mathrm{mM}),(4-6)$ the reaction was performed by adding $3 \mu \mathrm{L}$ $\mathrm{SCN}^{-}$solution $(50 \mathrm{mM})$ into the open $\mathrm{Fe}^{3+}$ liquid marble with a pipette, and (7-9) the optical detection of the product of $\mathrm{Fe}(\mathrm{SCN})^{2+}$ complex ions. b Optical absorption curves of magnetic liquid marbles containing $\mathrm{Fe}^{3+}$ and $\mathrm{Fe}(\mathrm{SCN})^{2+}$ solutions, respectively (a)

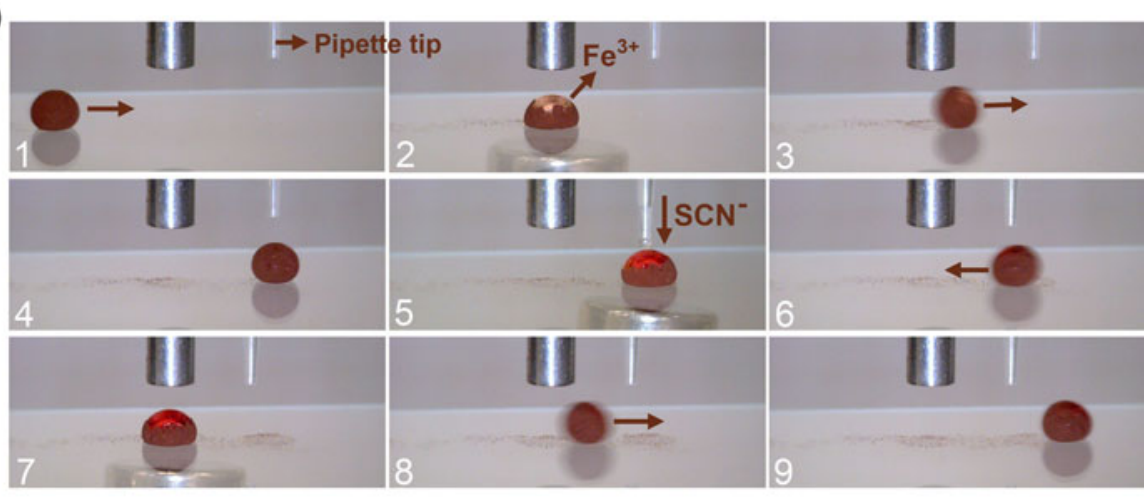

(b)

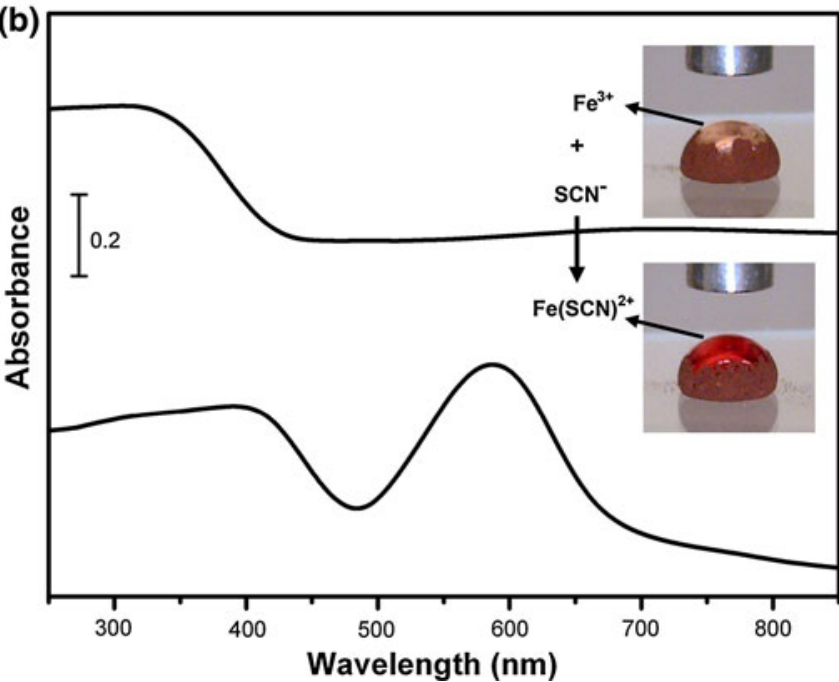

Figure 10a shows the optical absorbance curves of magnetic liquid marbles containing aqueous solutions of acid blue 25 with various concentrations. Over the range of concentrations examined $\left(0.2 \times 10^{-5}-1.2 \times 10^{-5} \mathrm{M}\right)$, the peak absorption increased with increasing the concentration. Figure $10 \mathrm{~b}$ shows the calibration plot of absorption as a function of the concentration, with a correlation coefficient of 0.9906 . Such linearity indicates the capability to conduct reliable quantitative optical measurements using this new technique based on magnetic liquid marbles.

In addition, a chemical reaction was performed by adding $\mathrm{SCN}^{-}$-containing solution to an opened magnetic 
liquid marble containing $\mathrm{Fe}^{3+}$ ions, followed by the optical detection. As shown in Fig. 11a, a magnetic liquid marble $(15 \mu \mathrm{L})$ containing $\mathrm{Fe}^{3+}$ solution $(10 \mathrm{mM})$ is moved to a defined location and then opened for optical detection (images 1-3). It was then moved to the position below a pipette tip and opened for the injection of $\mathrm{SCN}^{-}$solution ( $3 \mu \mathrm{L}, 50 \mathrm{mM}$ ) (images 4-5). After injection, the solution was observed to change from yellow $\left(\mathrm{Fe}^{3+}\right)$ to deep red $\left[\mathrm{Fe}(\mathrm{SCN})^{2+}\right]$. Finally, the liquid marble containing the product, $\mathrm{Fe}(\mathrm{SCN})^{2+}$ complex ions, was closed and moved for another optical detection, followed by being disposed (images 6-9). Figure 11b shows the optical absorption curves of magnetic liquid marbles containing $\mathrm{Fe}^{3+}$ and $\mathrm{Fe}(\mathrm{SCN})^{2+}$ solutions, respectively. It may form a new platform technology to use magnetic liquid marbles as a "smart" microreactor for chemical analysis or exploring new chemical reactions, especially when precious, explosive or highly toxic reagents are used and the products need to be stored without separation. Compared with the more conventional droplet-based microfluidics, such as water-inoil droplets or electrowetting-on-dielectrics (EWOD), the relatively low throughput can be improved using magnet arrays instead of a single magnet (Ohashi et al. 2007), and the automation of the processing steps can be realized by using electromagnetic field.

\section{Conclusions}

We have presented the manipulation of a magnetic liquid marble under an external magnetic field and calculated the maximum frictional force, the magnetic force required for actuating the liquid marbles, and the effective surface tension of the magnetic liquid marble, as well as the threshold volume for the transition from quasi-spherical to puddle-like shape. By taking advantage of the unique feature of being opened and closed reversibly, we have proven that the encapsulated droplets can be detected optically with a reflection-mode probe. Combining the open-close and optical detection also enables to probe chemical reactions taking place within liquid marbles. These remarkable features offer a simple yet powerful alternative to conventional discrete microfluidic systems and may have wide applications in biomedical and drug discovery.

Acknowledgments This work is supported by the Australian Research Council (ARC) under grant DP110101315. YZ thanks the Australian Postdoctoral (APD) fellowship support from ARC.

Open Access This article is distributed under the terms of the Creative Commons Attribution License which permits any use, distribution, and reproduction in any medium, provided the original author(s) and the source are credited.

\section{References}

Abdelgawad M, Wheeler AR (2009) The digital revolution: a new paradigm for microfluidics. Adv Mater 21:920

Abdelgawad M, Freire SLS, Yang H, Wheeler AR (2008) All-terrain droplet actuation. Lab Chip 8:672

Aussillous P, Quere D (2001) Liquid marbles. Nature 411:924

Bormashenko E (2011) Liquid marbles: properties and applications. Curr Opin Colloid Interface Sci 16:266

Bormashenko E, Pogreb R, Bormashenko Y, Musin A, Stein T (2008) New investigations on ferrofluidics: ferrofluidic marbles and magnetic-field-driven drops on superhydrophobic surfaces. Langmuir 24:12119

Callies M, Quere D (2005) On water repellency. Soft Matter 1:55

Dandan M, Erbil HY (2009) Evaporation rate of graphite liquid marbles: comparison with water droplets. Langmuir 25:8362

Dorvee JR, Derfus AM, Bhatia SN, Sailor MJ (2004) Manipulation of liquid droplets using amphiphilic, magnetic one-dimensional photonic crystal chaperones. Nat Mater 3:896

Egatz-Gomez A, Melle S, Garcia AA, Lindsay SA, Marquez M, Dominguez-Garcia P et al (2006) Discrete magnetic microfluidics. Appl Phys Lett 89:034106

Egatz-Gomez A, Schneider J, Aella P, Yang D, Dominguez-Garcia P, Lindsay $S$ et al (2007) Silicon nanowire and polyethylene superhydrophobic surfaces for discrete magnetic microfluidics. Appl Surf Sci 254:330

Feng L, Li SH, Li YS, Li HJ, Zhang LJ, Zhai J et al (2002) Superhydrophobic surfaces: from natural to artificial. Adv Mater 14:1857

Gao LC, McCarthy TJ (2007) Ionic liquid marbles. Langmuir 23:10445

Garcia AA, Egatz-Gomez A, Lindsay SA, Dominguez-Garcia P, Melle S, Marquez M et al (2007) Magnetic movement of biological fluid droplets. J Magn Magn Mater 311:238

Guo ZG, Zhou F, Hao JC, Liang YM, Liu WM, Huck WTS (2006) "Stick and slide" ferrofluidic droplets on superhydrophobic surfaces. Appl Phys Lett 89:081911

Guttenberg Z, Muller H, Habermuller H, Geisbauer A, Pipper J, Felbel J et al (2005) Planar chip device for PCR and hybridization with surface acoustic wave pump. Lab Chip 5:308

Hong L, Pan T (2011) Surface microfluidics fabricated by photopatternable superhydrophobic nanocomposite. Microfluidic Nanofluid 10:991

Huang C, Zhou Y, Jin Y, Zhou X, Tang Z, Guo X et al (2011) Preparation and characterization of temperature-responsive and magnetic nanomicelles. J Mater Chem 21:5660

Lehmann U, Vandevyver C, Parashar VK, Gijs MAM (2006) Dropletbased DNA purification in a magnetic lab-on-a-chip. Angew Chem Int Ed 45:3062

Lindsay S, Vazquez T, Egatz-Gomez A, Loyprasert S, Garcia AA, Wang J (2007) Discrete microfluidics with electrochemical detection. Analyst 132:412

McHale G, Newton MI (2011) Liquid marbles: principles and applications. Soft Matter 7:5473

McHale G, Shirtcliffe NJ, Newton MI (2004) Super-hydrophobic and super-wetting surfaces: analytical potential? Analyst 129:284

McHale G, Shirtcliffe NJ, Newton MI, Pyatt FB, Doerr SH (2007) Self-organization of hydrophobic soil and granular surfaces. Appl Phys Lett 90:054110

Mumm F, van Helvoort ATJ, Sikorski P (2009) Easy route to superhydrophobic copper-based wire-guided droplet microfluidic systems. ACS Nano 3:2647

Ohashi T, Kuyama H, Hanafusa N, Togawa Y (2007) A simple device using magnetic transportation for droplet-based PCR. Biomed Microdevices 9:695 
Park J, An K, Hwang Y, Park JG, Noh HJ, Kim JY et al (2004) Ultralarge-scale syntheses of monodisperse nanocrystals. Nat Mater 3:891

Quere D (1998) Drops at rest on a tilted plane. Langmuir 14:2213

Quere D, Aussillous P (2002) Non-stick droplets. Chem Eng Technol 25:925

Schneider J, Egatz-Gomez A, Melle S, Lindsay S, Dominguez-Garcia P, Rubio MA et al (2008) Motion of viscous drops on superhydrophobic surfaces due to magnetic gradients. Colloids Surf A 323:19

Srinivasan V, Pamula VK, Fair RB (2004) Droplet-based microfluidic lab-on-a-chip for glucose detection. Anal Chim Acta 507:145
Xue Y, Wang H, Zhao Y, Dai L, Feng L, Wang X et al (2010) Magnetic liquid marbles: a "precise" miniature reactor. Adv Mater 22:4814

Yoon J-Y, You JD (2008) Backscatttering particle immunoassays in wire-guide droplet manipulations. J Biomed Eng 2:15

Zhang K, Liang Q, Ma S, Mu X, Hu P, Wang Y et al (2009) On-chip manipulation of continuous picoliter-volume superparamagnetic droplets using a magnetic force. Lab Chip 9:2992

Zhao Y, Fang J, Wang H, Wang X, Lin T (2010) Magnetic liquid marbles: manipulation of liquid droplets using highly hydrophobic $\mathrm{Fe}_{3} \mathrm{O}_{4}$ nanoparticles. Adv Mater 22:707 